\title{
A SEGREGAÇÃO DO GÊNERO ACACIA (TOURN.) MILL., SOB O PONTO DE VISTA DA ANATOMIA DA MADEIRA DE ESPÉCIES NATIVAS E CULTIVADAS NO RIO GRANDE DO SUL ${ }^{1}$
}

\author{
JOSÉ NEWTON CARDOSO MARCHIORI² SIDINEI RODRIGUES DOS SANTOS ${ }^{3}$
}

\begin{abstract}
RESUMO
É analisada a segregação do gênero Acacia (Tourn.) Mill. (Fabaceae) sob o ponto de vista da anatomia da madeira, com base em 14 espécies nativas e cultivadas no Rio Grande do Sul, pertencentes, atualmente, a três gêneros distintos: Acacia, Senegalia e Vachellia. Foram reconhecidas diferenças estruturais suficientes para a identificação anatômica destes três gêneros.

Palavras-chave: Taxonomia da madeira, Acacia, Vachellia, Senegalia, Fabaceae, Leguminosae Mimosoideae.
\end{abstract}

\section{SUMMARY}

[The segregation of genus Acacia (Tourn.) Mill., according to wood anatomy of native and cultivated species in Rio Grande do Sul state, Brazil].

The segregation of Acacia (Tourn.) Mill. (Fabaceae) is anatomically examined, based on 14 native and cultivated species of wood from Rio Grande do Sul state (Brazil), actually positioned in three distinct genus: Acacia, Senegalia and Vachellia. Enough structural differences for the anatomical identification of these three genera were presently recognized.

Key words: Wood taxonomy, Acacia, Vachellia, Senegalia, Fabaceae, Leguminosae Mimosoideae.

\section{INTRODUÇÃO}

Com a recente segregação do gênero Acacia (Tourn.) Mill., as espécies americanas foram distribuídas entre os gêneros Vachellia Wight \& Arnott e Senegalia Raf., restando em Acacia apenas as espécies australianas introduzidas no continente. Em decorrência destas mudanças, o presente estudo da madeira de 14 espécies nativas e cultivadas no Rio Grande do Sul, pertencentes ao antigo gênero, visa, justamente, a examinar esta questão sob o ponto de vista da anatomia da madeira, buscando o reconhecimento de caracteres anatômicos de valor diagnóstico para os táxones envolvidos.

\footnotetext{
1 Recebido em 11-1-2011 e aceito para publicação em 236-2011.

${ }^{2}$ Engenheiro Florestal, Dr. Bolsista de Produtividade em Pesquisa (CNPq - Brasil). Professor Titular do Departamento de Ciências Florestais, Universidade Federal de Santa Maria. 97.015-663, Santa Maria, RS, Brasil. marchiori@pq.cnpq.br

${ }^{3}$ Biólogo, bolsista (CNPq - Brasil), doutorando do Programa de Pós Graduação em Engenharia Florestal, Universidade Federal de Santa Maria. sthurt.bio@gmail.com
}

\section{REVISÃO DE LITERATURA}

O gênero Acacia foi estabelecido por Phillip Miller em 1754, com base em nome proposto por Tournefort (1694). O termo origina-se do grego akakia, um substantivo que se traduz por ponta ou fio (Miller, 1969) e alude à presença conspícua de espinhos no caule e ramos de muitas de suas espécies.

$\mathrm{O}$ antigo gênero estava bem representado nas regiões tropicais e subtropicais da América, África, Ásia e Austrália, faltando, apenas, na Europa e Nova Zelândia (Burkart, 1952). De um total de aproximadamente 1200 espécies, mais da metade (cerca de 700), todavia, era nativa da Austrália (Pedley, 1986).

Com base em caracteres do androceu, George Bentham - o mais importante Leguminólogo do século XIX - criou a tribo Acacieae em 1842, juntamente com outras cinco da sub-ordem Mimoseae, táxon de extensão correspondente à atual sub-família Mimosoideae. Único em sua tribo, o gênero Acacia, por sua vez, foi classificado em seis séries fundamentais, com base em caracteres taxonômicos clássicos da morfologia 
floral e aparelho vegetativo: Phyllodineae, Botryocephalae, Pulchellae, Gummiferae, Vulgares e Filicinae.

Caracterizada pela redução das folhas a filódios, a série Phyllodineae compreendia, originalmente, 204 espécies da Austrália e ilhas do Oceano Pacífico (Bentham, 1842). Em publicação posterior, Bentham (1875) relacionou 227 espécies para a mesma série, distribuindoas em oito subséries.

Da mesma região geográfica, a série Botryocephalae foi criada para abranger espécies inermes, de folhas bipinadas e capítulos racemosos. Composto, originalmente, por 13 espécies (Bentham, 1842), o grupo foi reduzido a 10 na revisão de 1875 .

De distribuição australásica, a Série Pulchellae reunia, inicialmente, 11 espécies (Bentham, 1842) inermes ou armadas de espinhos axilares, com folhas bipinadas e flores em capítulos globosos ou espigas cilíndricas, dispostas na axila de folhas ou em racemos terminais pedunculados. Na revisão do grupo, foram reconhecidos apenas oito binômicos (Bentham, 1875).

Formada por 48 espécies americanas, da África e Ásia (Bentham, 1842), a série Gummiferae foi criada para abranger as acácias desprovidas de acúleos, mas com estípulas espinescentes e folhas bipinadas. Na revisão do grupo, Bentham (1875) ampliou-o para 60 espécies, distribuídas em três subséries.

A série Vulgares compreendia, originalmente, 53 espécies (Bentham, 1842) de árvores e arbustos com folhas bipinadas, estípulas não espinescentes, pecíolo glandulífero e acúleos infraestipulares esparsos ou ausentes, distribuídas em 5 sub-séries. Na segunda revisão, este número aumentou para $75 \mathrm{e}$ as espécies foram reorganizadas em 4 sub-séries (Bentham, 1875).

A sexta série de Acacia, denominada Filicinae, compunha-se, inicialmente, de 11 espécies americanas de arbustos inermes, com folhas bipinadas e pecíolo eglanduloso (Bentham, 1842). Na revisão de 1875 , o autor conservou apenas duas espécies "da região me- xicana" nesta série botânica, reconhecida como a mais arcaica do gênero (Guinet \& Vassal, 1978), por seus numerosos caracteres indiferenciados.

De longa vigência, a classificação de George Bentham dominou na literatura taxonômica por mais de um século, até o reordenamento proposto por Vassal (1963, 1972a,b), com base em caracteres filéticos da morfologia da semente e plântulas. $\mathrm{O}$ gênero monotípico Faidherbia ( $F$. albida (Del.) A. Chev.) foi, então, restaurado e as demais espécies de Acacia (Tourn.) Mill. acabaram segregadas em três subgêneros: Aculeiferum, Acacia e Heterophyllum. O primeiro destes (Aculeiferum), baseia-se nas séries Vulgares e Filicinae de Bentham; o subgênero Acacia abrange as espécies da série Gummiferae Benth., com exceção de Acacia albida Del.; e Heterophyllum reúne as espécies australásicas das séries Phyllodineae, Botryocephalae e Pulchellae, de Bentham.

Em 1986, Pedley transferiu as espécies do subgênero Heterophyllum Vassal para o gênero Racosperma, levando, por conseguinte, à renomeação das espécies australianas cultivadas no sul do Brasil: Racosperma dealbatum (Link) Pedley (antiga Acacia dealbata Link); Racosperma decurrens (Willd.) Pedley (Acacia decurrens Willd.); Racosperma mearnsii (De Wild.) Pedley (Acacia mearnsii De Wild.); Racosperma melanoxylon (R. Br.) Pedley (Acacia melanoxylon R. Br.); e Racosperma podalyriifolium (G. Don) Pedley (Acacia podalyriifolia G. Don). Uma sinopse deste gênero botânico veio a lume posteriormente (Pedley, 2003).

A proposta de Pedley (1986) teve curta validade, pois estudos morfológicos e moleculares comprovaram que o antigo gênero Acacia, por ser polifilético, necessitava de uma divisão mais radical (Maslin et al. 2003).

Em 30 de Julho de 2005, o Congresso Internacional de Botânica de Viena endossou a proposta de Orchard \& Maslin (2003), preservando o nome genérico para o grupo mais numeroso (Phyllodineae), mediante retipificacão. Des- 
te modo, a espécie-tipo do gênero foi transferida de Acacia nilotica (L.) Delile ${ }^{4}$, originária da África, para a espécie australiana Acacia penninervis Sieber ex DC. (McNeil et al., 2005). O gênero Acacia, deste modo, compreende atualmente 948 espécies australianas, além de outras dez da Ásia tropical, sete de ilhas do Oceano Pacífico e uma ou duas de Madagascar. Das espécies australianas, apenas sete foram transferidas para Vachellia e duas para Senegalia (Maslin et al., 2003).

No caso das espécies americanas, elas foram transferidas para os gêneros Vachellia Wight \& Arnott e Senegalia Raf. (Siegler \& Ebinger, 2005). O primeiro deles, que abrange as antigas acácias do subgênero Acacia (ou série Gummiferae, de Bentham), reúne 159 espécies nas três Américas e, aproximadamente, 52 táxones infraespecíficos. Baseado no antigo subgênero Aculeiferum Vassal, o novo gênero Senegalia, por sua vez, compreende 53 espécies no mesmo continente e 17 táxones infraespecíficos (Rico-Arce, 2007).

No tocante à anatomia da madeira, cabe salientar, de início, que a literatura ainda é relativamente escassa, face à importância econômica e extensão do antigo gênero Acacia. Record \& Hess (1949), por exemplo, um dos clássicos da literatura anatômica, não fez nenhuma referência ao mesmo, apesar das numerosas espécies nativas no continente americano.

Cozzo (1951), em importante estudo sobre madeiras argentinas de Leguminosas Mimosoídeas e Cesalpinioídeas, não encontrou diferenças anatômicas relevantes nas 13 espécies de Acacia por ele investigadas, motivo pelo qual o gênero foi definido como "estruturalmente homogêneo". O autor relacionou uma série de detalhes estruturais para as distintas espécies, fornecendo, inclusive, uma chave dicotômica para a identificação das mesmas.

Em estudo de 37 acácias sul-africanas, Robbertse et al. (1980) também observaram

${ }^{4}$ Antiga espécie tipo, Acacia nilotica pertencia à série Gummiferae Benth. (ou subgênero Acacia Vassal). pequena variação na estrutura anatômica. A dimensão dos raios e, com menor importância, a presença de anéis de crescimento, número de poros $/ \mathrm{mm}^{2} \mathrm{e}$ a distribuição do parênquima axial foram os principais caracteres utilizados na distinção de subgêneros. Todas as 14 espécies examinadas do subgênero Aculeiferum tinham raios estreitos (2-5-seriados), em contraste com as 23 espécies do subgênero Acacia, distintas pelos raios mais largos (4-14-seriados).

Ao contrário dos dois trabalhos acima comentados, Marchiori (1990) observou diferenças "bastante expressivas" em estudo de 15 espécies nativas e cultivadas no Rio Grande do Sul, comparáveis às "registradas entre espécies de diferentes gêneros e tribos de Mimosoideae". Esta variabilidade estrutural levou o autor a reconhecer três grupos distintos no tocante à anatomia da madeira, correspondentes aos subgêneros criados por Vassal (1972b).

\section{MATERIAL ESTUDADO}

O presente estudo inclui 14 espécies nativas e cultivadas no Rio Grande do Sul, pertencentes ao antigo gênero Acacia (Tourn.) Mill. A relação dos nomes científicos atualmente válidos e dos respectivos binômios reduzidos à sinonímia é apresentada na Tabela 1, juntamente com a seção botânica (Bentham, 1842, 1875) e subgênero (Vassal (1972a, b) aos quais estavam inseridas as distintas espécies, antes da segregação em análise.

As 14 espécies em estudo foram previamente publicadas nos seguintes artigos individuais, listados segundo ordem alfabética das espécies e nomenclatura em vigor: Acacia dealbata (Marchiori, 2011b); Acacia decurrens (Marchiori, 2011a); Acacia longifolia (Illana \& Marchiori, 1980); Acacia mearnsii (Costa \& Marchiori, 1980); Acacia melanoxylon (Marchiori, 2009); Acacia podalyriifolia (Marchiori, 2010a); Senegalia bonariensis (Marchiori, 1980, 1996); Senegalia nitidifolia (Marchiori, 1991); Senegalia recurva (Marchiori, 1982); Senegalia tucumanensis (Marchiori, 1980, 1994); Senegalia velutina 
TABELA 1 - Espécies estudadas, com as respectivas séries botânicas e subgêneros.

\begin{tabular}{|c|c|c|}
\hline Nome científico & Série & Subgênero \\
\hline $\begin{array}{l}\text { Acacia dealbata Link } \\
\text { [Acacia dealbata Link] }\end{array}$ & Botryocephalae & Heterophyllum \\
\hline $\begin{array}{l}\text { Acacia decurrens Willd. } \\
\text { [Acacia decurrens (Wendl.) Willd.] }\end{array}$ & Botryocephalae & Heterophyllum \\
\hline $\begin{array}{l}\text { Acacia longifolia (Andrews) Willd. } \\
\text { [Acacia longifolia (Andrews) Willd.] }\end{array}$ & Phyllodineae & Heterophyllum \\
\hline $\begin{array}{l}\text { Acacia mearnsii De Wild. } \\
\text { [Acacia mearnsii De Wild.] }\end{array}$ & Botryocephalae & Heterophyllum \\
\hline $\begin{array}{l}\text { Acacia melanoxylon } \mathrm{R} . \mathrm{Br} \text {. } \\
\text { [Acacia melanoxylon } \mathrm{R} \text {. Br.] }\end{array}$ & Phyllodineae & Heterophyllum \\
\hline $\begin{array}{l}\text { Acacia podalyriifolia G. Don } \\
\text { [Acacia podalyriifolia G. Don] }\end{array}$ & Phyllodineae & Heterophyllum \\
\hline $\begin{array}{l}\text { Senegalia bonariensis (Gillies ex Hook. \& Arn.) Seigler \& Ebinger } \\
\text { [Acacia bonariensis Gillies ex Hook. \& Arn.] }\end{array}$ & Vulgares & Aculeiferum \\
\hline $\begin{array}{l}\text { Senegalia nitidifolia (Speg.) Seigler \& Ebinger } \\
\text { [Acacia nitidifolia Speg.] }\end{array}$ & Vulgares & Aculeiferum \\
\hline $\begin{array}{l}\text { Senegalia recurva (Benth.) Seigler \& Ebinger } \\
\text { [Acacia recurva Benth.] }\end{array}$ & Vulgares & Aculeiferum \\
\hline $\begin{array}{l}\text { Senegalia tucumanensis (Griseb.) Seigler \& Ebinger } \\
\text { [Acacia tucumanensis Griseb.] }\end{array}$ & Vulgares & Aculeiferum \\
\hline $\begin{array}{l}\text { Senegalia velutina (DC.) Seigler \& Ebinger } \\
\text { [Acacia velutina } \text { DC.] }\end{array}$ & Vulgares & Aculeiferum \\
\hline $\begin{array}{l}\text { Vachellia caven (Molina) Seigler \& Ebinger } \\
\text { [Acacia caven (Molina) Molina] }\end{array}$ & Gummiferae & Acacia \\
\hline $\begin{array}{l}\text { Vachellia farnesiana (L.) Wight \& Arn. } \\
\text { [Acacia farnesiana (L.) Willd.] }\end{array}$ & Gummiferae & Acacia \\
\hline $\begin{array}{l}\text { Vachellia ibirocayensis (Marchiori) Deble \& Marchiori } \\
\text { [Acacia ibirocayensis Marchiori] }\end{array}$ & Gummiferae & Acacia \\
\hline
\end{tabular}

Os binômios entre colchetes, constantes em obras mais antigos, caíram em sinonímia. Observar que as espécies australianas não sofreram alteração, neste sentido. 
(Marchiori, 1995); Vachellia caven (Marchiori, 1980, 1992; Marchiori et al., 2009); Vachellia farnesiana (Marchiori, 2010b) e Vachellia ibirocayensis (Marchiori, 1993). A Tabela 2, que resume os principais caracteres anatômicos de valor diagnóstico para a distinção dos três gêneros envolvidos, foi elaborada, justamente, a partir de caracteres anatômicos colhidos nas publicações acima relacionadas.

\section{ANÁLISE ANATÔMICO-TAXONÔMICA}

Os caracteres anatômicos comuns às 14 espécies investigadas, tais como elementos vasculares muito curtos a curtos, placas de perfuração simples, pontoado intervascular alterno, pontoações ornamentadas de diâmetro pequeno a médio, parênquima paratraqueal, raios homogêneos e fibras libriformes, são de ampla em Leguminosas (Fabaceae), de acordo com

TABELA 2 - Caracteres anatômicos de espécies de Acacia, Senegalia e Vachellia, nativas e cultivadas no Rio Grande do Sul.

\begin{tabular}{|c|c|c|c|c|c|c|c|c|}
\hline Espécie & Fs & Fns & $\mathrm{PAa}$ & PAe & $\operatorname{Re}$ & $\mathbf{R I}$ & $\mathrm{Cg}$ & $\mathrm{Cp}$ \\
\hline Acacia dealbata & & $x$ & & $x$ & $x$ & & & $x$ \\
\hline Acacia decurrens & & $x$ & & $x$ & $x$ & & & $x$ \\
\hline Acacia longifolia & & $x$ & & $x$ & $x$ & & & $x$ \\
\hline Acacia mearnsii & & $x$ & & $x$ & $x$ & & & $x$ \\
\hline Acacia melanoxylon & & $x$ & & $x$ & $\mathrm{x}$ & & & $x$ \\
\hline Acacia podalyriifolia & & $x$ & & $x$ & $x$ & & & $x$ \\
\hline Senegalia bonariensis & $x$ & & & $x$ & $x$ & & & $x$ \\
\hline Senegalia nitidifolia & $x$ & & & $x$ & $x$ & & & $x$ \\
\hline Senegalia recurva & $x$ & & & $x$ & $x$ & & & $x$ \\
\hline Senegalia tucumanensis & $x$ & & & $x$ & $x$ & & & $x$ \\
\hline Senegalia velutina & $x$ & & & $x$ & $x$ & & & $x$ \\
\hline Vachellia caven & & $x$ & $x$ & & & $x$ & $x$ & \\
\hline Vachellia farnesiana & & $x$ & $x$ & & & $x$ & $x$ & \\
\hline Vachellia ibirocayensis & & $x$ & $x$ & & $x$ & & & $x$ \\
\hline
\end{tabular}

Fs $=$ Fibras septadas. Fns $=$ Fibras não septadas. $\mathrm{PAa}=$ Parênquima axial abundante $(>25 \%)$. PAe $=$ Parênquima axial escasso $(<20 \%) . R e=$ Raios estreitos $(1-4$-seriados $) . R 1=$ com raios largos ou conspícuos $(+$ de 6 células de largura). $\mathrm{Cg}=$ Cristais grandes $(>60 \mu \mathrm{m})$, presentes. $\mathrm{Cp}=$ Cristais pequenos $(<25 \mu \mathrm{m})$, apenas. 

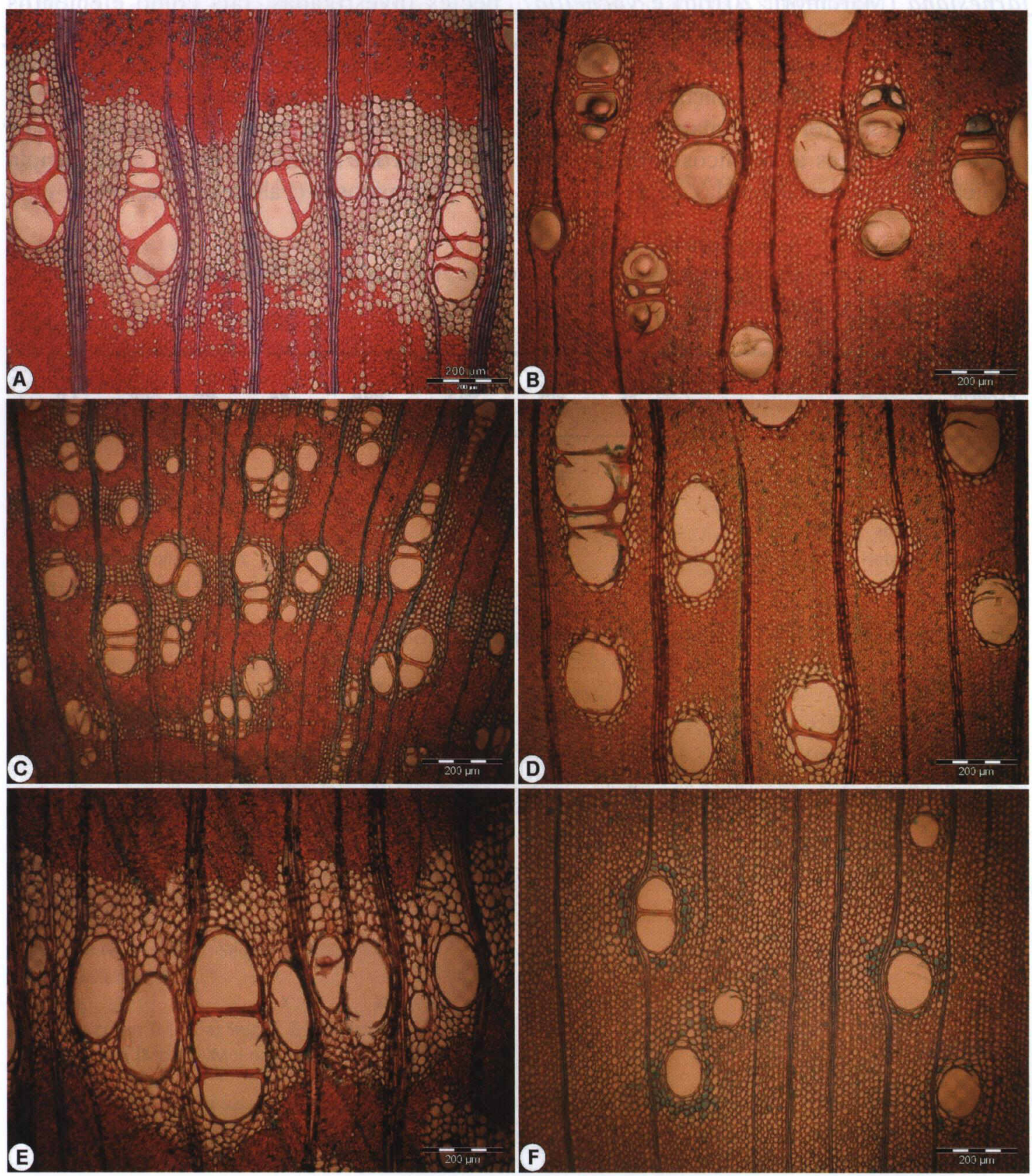

FIGURA 1 - O parênquima paratraqueal, em seis espécies (corte transversal). A, C, E - Vachellia caven, Vachellia ibirocayensis e Vachellia farnesiana, respectivamente, madeiras de parênquima axial abundante ( $>25 \%)$, em faixas tangenciais irregulares. B, D, F-Acacia decurrens, Acacia dealbata e Senegalia recurva, respectivamente, espécies de parênquima axial relativamente escasso $(<25 \%)$ e em arranjo paratraqueal vasicêntrico. 
Metcalfe \& Chalk (1972), Record \& Hess (1949), Cozzo (1950, 1951) e Tortorelli (1956), entre outros autores. A ausência de estratificação, bem como os raios compostos inteiramente por células procumbentes de seção pequena, em plano tangencial, vincula as espécies em estudo à subfamília Mimosoideae, no conjunto das Leguminosas (Fabaceae), de acordo com Baretta-Kuipers (1981). Embora valiosos para a identificação anatômica de Fabaceae e Mimosoideae, respectivamente, este conjunto de caracteres apresenta ampla ocorrência taxonômica, motivo pelo qual carece de importância no presente estudo, voltado ao reconhecimento de diferenças anatômicas intergenéricas.

Basta um rápido passar de olhos na Tabela 2 , todavia, para se contestar a opinião de Cozzo (1951) sobre a homogeneidade estrutural do xilema secundário no antigo gênero Acacia. De fato, ao contrário do afirmado pelo anatomista argentino, os dados ali reunidos comprovam a existência de diferenças anatômicas consideráveis entre as espécies em estudo, concordando, neste sentido, com o observado por Robbertse et al. (1980), em estudo de 37 espécies sul-africanas. $\mathrm{O}$ exame mais detido da mesma Tabela, além disso, permite o reconhecimento de três grupos distintos sob o ponto de vista da anatomia da madeira, os quais coincidem com os gêneros criados pela segregação de Acacia (Tourn.) Mill. (Acacia, Senegalia, Vachellia), bem como com os antigos subgêneros (Heterophyllum, Aculeiferum, Acacia) e séries botânicas (Phyllodineae + Botryocephalae, Vulgares, Gummiferae) do mesmo.

Vachellia caven, V. farnesiana e $V$. ibirocayensis constituem um grupo anatomicamente distinto, por associarem fibras não septadas (Figura 2B) a parênquima axial abundante (Figura 1A, C, E), composto por séries de 2, no máximo 3 células de altura (Figura 3A, C, E). Segregadas do gênero Acacia, estas três espécies pertenciam ao subgênero Acacia, de acordo com Vassal (1972a, b), ou à série Gummiferae, segundo Bentham $(1842,1875)$.
Vachellia caven e V. farnesiana separam-se das demais espécies, ainda, por terem raios conspícuos (Figura 3A, C), com alta percentagem de multisseriados, ou seja, de raios com mais de 4 células de largura (Marchiori, 1992 e 2010 b, respectivamente). Foi com base neste caráter, aliás, que Robbertse et al. (1980) distinguiram os antigos subgêneros Acacia (atual gênero Vachellia) e Aculeiferum (atual gênero Senegalia). No caso de Vachellia ibirocayensis (Figura 3E), a presença de raios mais estreitos pode ser debitada ao pequeno diâmetro do caule no material examinado, aspecto sugestivo de pedomorfose, segundo Carlquist (1975).

As espécies de Senegalia Raf., por sua vez, separam-se das madeiras de Vachellia, anteriormente analisadas, bem como das seis espécies australianas de Acacia, pela presença conspícua de septos em suas fibras (Tabela 2). Este caráter anatômico, presentemente ilustrado com Senegalia tucumanensis (Figura 2A), Senegalia bonariensis (Figura 2C) e Senegalia recurva (Figura 2E), também se observa nas madeiras de Senegalia nitidifolia e Senegalia velutina, de acordo com Marchiori (1991) e Marchiori (1995), respectivamente, confirmando o valor diagnóstico do caráter para o gênero.

A estrutura anatômica da madeira das seis espécies australianas de Acacia, presentemente estudadas (A. dealbata, A. decurrens, A. longifolia, A. mearnsii, A. melanoxylon e A. podalyriifolia), por sua vez, compõem um grupo homogêneo e distinto, no presente estudo. Comparado às espécies de Senegalia, anteriormente comentadas, o grupo separa-se, facilmente, por ter fibras não septadas (Tabela 2; Figura 2D, F). No confronto com as três espécies do gênero Vachellia, estas seis acácias australianas distinguem-se, sobretudo, pelo parênquima relativamente escasso, inferior a $20 \%$ (Tabela 2; Figura 1B, D), composto por séries de 2 a 4 células (Figura 3B,D). As três espécies de Vachellia apresentam, distintamente, parênquima axial abundante, superior a $25 \%$ do volume da madeira (Tabela 2), contrastado com o tecido fibroso em plano transversal (Figuras 

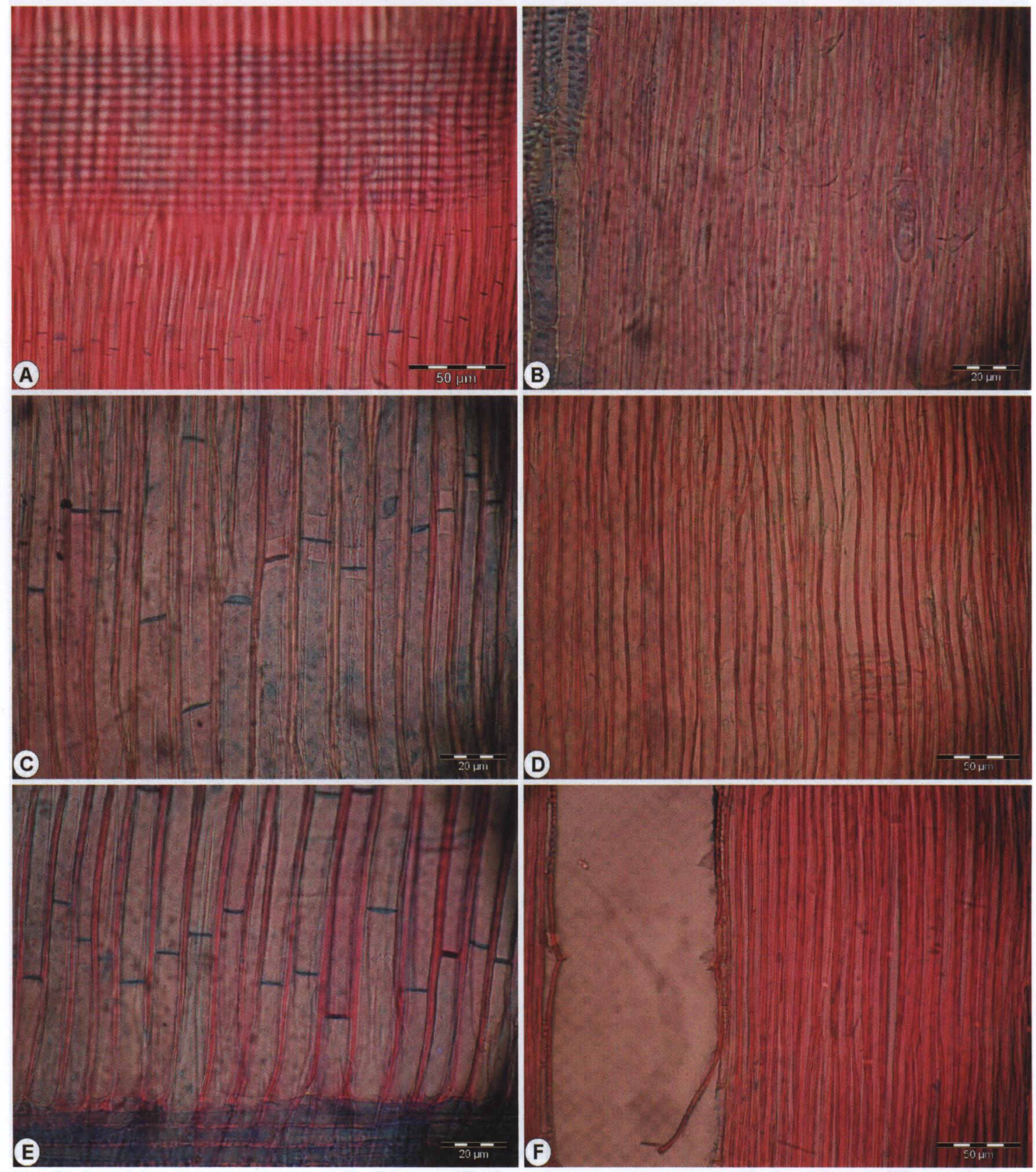

FIGURA 2 - A natureza das fibras, nos três gêneros em estudo (seção longitudinal radial). A, C, E-Espécies com fibras septadas (Senegalia tucumanensis, Senegalia bonariensis e Senegalia recurva, respectivamente). B, D, F - Espécies de fibras não septadas (Vachellia ibirocayensis, Acacia podalyriifolia e Acacia longifolia, respectivamente). 

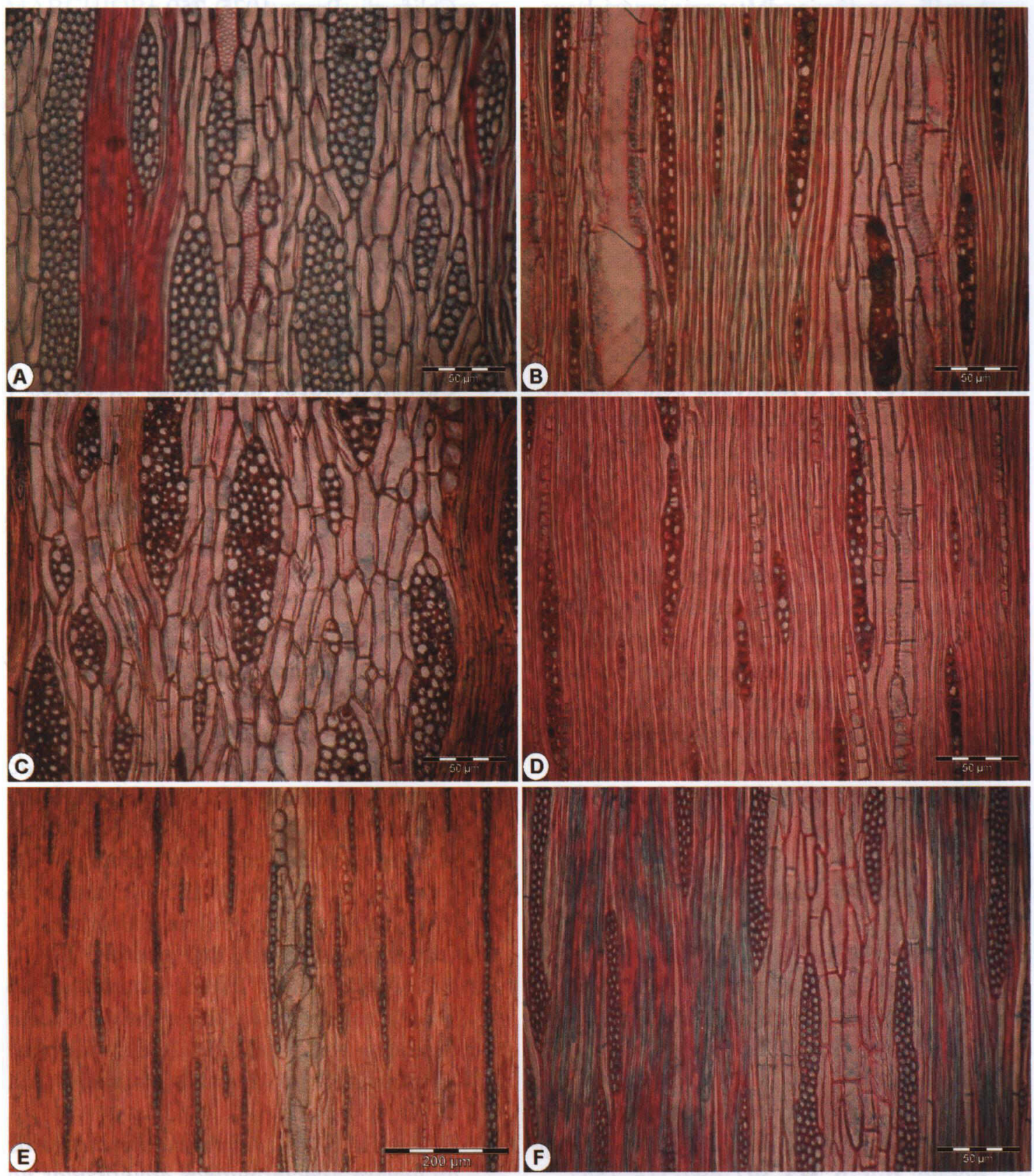

FIGURA 3 - Raios e séries de parênquima axial, em seção longitudinal tangencial. A, C - Raios conspícuos e séries parenquimáticas de 2 (raro 3) células (Vachellia caven e Vachellia farnesiana, respectivamente). B, D, F-Raios estreitos e séries parenquimáticas com 2-4 células (Acacia podalyriifolia, Acacia longifolia e Senegalia recurva, respectivamente). E - Vachellia ibirocayensis, madeira de raios estreitos, com séries de 2 (raro 3) células de altura. 
1A,C.E) e com séries de duas (raramente três) células de altura (Figuras 3A, C, E).

A análise anatômica destas seis espécies australianas, por outro lado, não aponta diferenças significativas entre as madeiras de Acacia dealbata, Acacia decurrens e Acacia mearnsii, pertencentes à antiga série Botryocephalae, $\mathrm{e}$ as madeiras de Acacia longifolia, Acacia melanoxylon e Acacia podalyriifolia, das Phyllodineae, segundo Bentham $(1842,1875)$. Mostra-se muito adequada, portanto, sob o ponto de vista da anatomia da madeira, a reunião destes táxones num mesmo subgênero (Heterophyllum), como proposto inicialmente por Vassal (1972), e ratificada pela atual circunscrição do gênero Acacia, definida no Congresso Internacional de Botânica de Viena, em 2005.

\section{CONCLUSÃO}

O estudo anatômico de 14 espécies de madeiras nativas e cultivadas no Rio Grande do Sul reforça a segregação do antigo gênero Acacia, promovida no Congresso Internacional de Botânica de Viena, em 2005, uma vez que os atuais gêneros Acacia, Senegalia e Vacchellia são perfeitamente distintos no tocante à estrutura do lenho.

\section{REFERÊNCIAS BIBLIOGRÁFICAS}

BARETTA-KUIPERS, T. The wood structure of leguminous tribes: their characterization by ray and parenchyma features. For. Prod. Abstr., v. 3, n. 8, p. 1784, 1980. (Resumo).

BENTHAM, G. Notes on Mimoseae, with a synopsis of species. The London Journal of Botany, n. 1, p. 318-392, 494-518, 1842.

BENTHAM, G. Revision of the sub-order Mimoseae. Trans. Linn. Soc. London, n. 30, p. 335-664, 1875.

BURKART, A. Las leguminosas argentinas silvestres y cultivadas. Buenos Aires: ACME Agency, 1952. 569 p.
CARLQUIST, S. Ecological strategies of xylem evolution. Berkeley: University of California Press, 1975. 259 p.

COSTA, A.F. da; MARCHIORI, J.N.C. Estudo anatômico da madeira de Acacia mearnsii De Willd. In: Anais do IV Congresso Florestal Estadual, Nova Prata, Rio Grande do Sul, 1980. p. 237-245.

COZZO, D. Anatomia del leño secundario de las Leguminosas Papilionoideas argentinas. Rev. Inst. Nac. Investig. Ci. Nat. Ci. Bot., v. 1, n. 7, p. 223-361, 1950.

COZZO, D. Anatomia del leño secundario de las Leguminosas Mimosoideas y Caesalpinoideas argentinas silvestres y cultivadas. Rev. Inst. Nac. Invest. Ci. Nat. Ci. Bot., v.2, n. 2, p. 63-290, 1951.

GUINET, P.; VASSAL, J. Hypotheses on differentiation of the major groups in the genus Acacia (Leguminosae). Kew Bulletin, v. 32 , p. $509-527,1978$.

ILLANA, H.A.; MARCHIORI, J.N.C. Estudo anatômico do xilema secundário de Acacia longifolia (Andr.) Willd. In: Anais do IV Congresso Florestal Estadual. Nova Prata, Rio Grande do Sul, 1980. p. 207-215.

MARCHIORI, J.N.C. Estudo anatômico do xilema secundário e da casca de algumas espécies dos gêneros Acacia e Mimosa, nativas no Estado do Rio Grande do Sul. Curitiba: UFPR, 1980. 185 f. Dissertação (Mestrado em Engenharia Florestal Universidade Federal do Paraná).

MARCHIORI, J.N.C. Estudo anatômico do xilema secundário e da casca de Acacia recurva Benth. (Leguminosae Mimosoideae). Ciência e Natura, Santa Maria, n. 4, p. 95-105, 1982.

MARCHIORI, J.N.C. Anatomia das madeiras do gênero Acacia, nativas e cultivadas no estado do Rio Grande do Sul. Curitiba: UFPR, 1990. $226 \mathrm{f}$. Tese (Doutorado em Engenharia Florestal - Universidade Federal do Paraná).

MARCHIORI, J.N.C. Anatomia da madeira de Acacia nitidifolia Speg. (Leguminosae 
Mimosoideae). Ciência Florestal, Santa Maria, v. 1, n. 1, p. 46-63, 1991.

MARCHIORI, J.N.C. Anatomia da madeira e casca do espinilho, Acacia caven (Mol.) Mol. Ciência Florestal, Santa Maria, v.2, n. 1, p. 27-47, 1992.

MARCHIORI, J.N.C. Estudo anatômico do xilema secundário de Acacia ibirocayensis Marchiori (Leguminosae Mimosoideae). Ciência e Natura, Santa Maria, n. 15, p. 149159, 1993.

MARCHIORI, J.N.C. Anatomia da madeira e casca de Acacia tucumanensis Gris. Ciência e Natura, Santa Maria, n. 16, p. 85-104, 1994.

MARCHIORI, J.N.C. Anatomia da madeira e casca de Acacia velutina DC. Ciência e Natura, Santa Maria, n. 17, p. 99-114, 1995.

MARCHIORI, J.N.C. Anatomia da madeira de Acacia bonariensis Gill. ex Hook. et Arn. Ciência Rural, Santa Maria, v. 26, n. 2, p. 209-216, 1996.

MARCHIORI, J.N.C. Anatomia da madeira de Acacia melanoxylon R. Br. Balduinia, Santa Maria, n. 18, p. 26-32, 2009.

MARCHIORI, J.N.C. Anatomia da madeira de Acacia podalyriaefolia A. Cunn. Balduinia, Santa Maria, n. 20, p. 10-15, 2010a.

MARCHIORI, J.N.C. Anatomia da madeira de Vachellia farnesiana (L.) Wight \& Arn. Balduinia, Santa Maria, n. 22, p. 9-14, 2010b.

MARCHIORI, J.N.C. Anatomia da madeira de Acacia decurrens Willd. Balduinia, Santa Maria, n. 26, p. 1-7, 2011a.

MARCHIORI, J.N.C. Anatomia da madeira de Acacia dealbata Link. Balduinia, Santa Maria, n. 29, p. 21-27, 2011 b.

MARCHIORI, J.N.C.; MUÑIZ, G.I.B. de; SANTOS, S.R. dos. Madeiras do Rio Grande do Sul. 1 - Descrição microscópica de 33 espécies nativas. Santa Maria: [s.n.], 2009. 80 p.
MASLIN, B.R.; MILLER, J.T.; SEIGLER, D.S. Overview of the generic status of Acacia (Leguminosae: Mimosoideae). Australian Systematic Botany, v. 16, p. 1-18, 2003.

McNEILL, J.; STUESSY, T.F.; TURLAND, N,J.; MÖRANDL, E. XVII International Botanical Congress: preliminary mail vote and report of Congress action on nomenclature proposals. Taxon, v. 54, n. 4, p. 1057-1064, 2005.

METCALFE, C.R.; CHALK, L. Anatomy of the Dicotyldeons. Oxford: Clarendon Press, 1972. $1500 \mathrm{p}$.

MILLER, P. The gardeners dictionary. New York: J. Cramer, 1969. 1582 p. (Abridged ed. 1754; Historia Naturalis Classica, LXXII).

ORCHARD, A.E.; MASLIN, B.R. Proposal to conserve the name Acacia (Leguminosae: Mimosoideae) with a conserved type. Taxon, v. 52, p. 362-363, 2003.

PEDLEY, L. Derivation and dispersal of Acacia (Leguminosae), with particular reference to Australia, and the recognition of Senegalia and Racosperma. Bot. J. Linn. Soc., Londres, n. 92, p. 219-254, 1986.

PEDLEY, L. Synopsis of Racosperma. Austrobaileya, v. 6, p. 445-496, 2003.

RECORD, S.J.; HESS, R.W. Timbers of the New World. New Haven: Yale University Press, 1949. $640 \mathrm{p}$.

RICO-ARCE, M. de L. American species of Acacia. México: Comisión Nacional para el Conocimiento y Uso de la Biodiversidad (CONABIO), 2007. 207 p.

ROBBERTSE, P.J.; VENTER, G.; RENSBURG, H.J. van. The wood anatomy of the south African Acacias. IAWA Bulletin, v. 1, n. 3, p. 93-103, 1980.

SEIGLER, D.S.; EBINGER, J.E. New combination in the genus Vachellia (Fabaceae: Mimosoideae) from the New World. Phytologia, v. 87, n. 3, p. 139-178, 2005 (publ. 2006). 
TORTORELLI, L.A. Maderas y bosques argentinos. Buenos Aires: ACME, 1956. $910 \mathrm{p}$.

TOURNEFORT, P. Elemens de Botanique ou Methode pour connoître les plantes. Paris: Imprimerie Royale, 1694. 379 p.

VASSAL, J. Intérêt taxonomique de la morphologie des graines dans le genre Acacia. Bull. de la Societé d'Histoire Naturel, Toulouse, v. 98 , n. 3-4, p. 341371, 1963.
VASSAL, J. Ontogenetic and seed research applied to the morphological, taxonomical and phylogenetic study of the genus Acacia. Travaux Lab. For. Toulouse, v. 1, n., 8, 1972a. $125 \mathrm{p}$.

VASSAL, J. Apport des recherches ontogénetiques et séminologiques à l'étude morphologique, taxonomique et phylogénique du genre Acacia. Bull. Soc.Hist. Nat., Toulouse, v. 108, p. 125-247, 1972b. 